\title{
The research and realization of a new iron ladle tracking system
}

\author{
Sheng Yin, Ming Yang, Chen Wang, Rong Luo \\ Center Of Enterprise Information Researching, Chongqing University of Posts and \\ Telecommunications
}

Keywords: Iron package tracking, RFID, steel production.

\begin{abstract}
Iron ladles tracking is the basis of BF-BOF iron production scheduling and is very important for the close convergence between the production of ironworks and steelworks. Recently in most of the domestic steel plants, the tracking of iron package is done by humans, which is unable to be timely access to the production conditions of online, and offline and cause the slow convergence in steel production and low productivity.
\end{abstract}

\section{Introduction}

Along with the development of Internet technology, the manufacturing industry has made a tremendous role, especially the RFID (radio frequency identification) technology as the core of the Internet of things, and the combination of manufacturing information system applications, can effectively achieve the manufacturing enterprise informatization and industrialization of perfect fusion, recognized by the height of the manufacturing enterprise, for enterprise to bring the efficiency of operation efficiency and management benefit at the same time, what is more important is that it can greatly improve enterprise comprehensive competitiveness. This article mainly research about steel iron package tracking management system of the new process which is based on RFID technology oriented "a pot of" ,the main purpose is to optimize the iron Ou De Tie packet scheduling, improve the utilization rate of iron package and reduce the time of the iron packets waiting in the station operation, so as to improve the package of iron from the blast furnace in the steelmaking process of converter production rhythm, reduce iron problem of temperature drop too much, and good for improving the production efficiency and reduce costs.

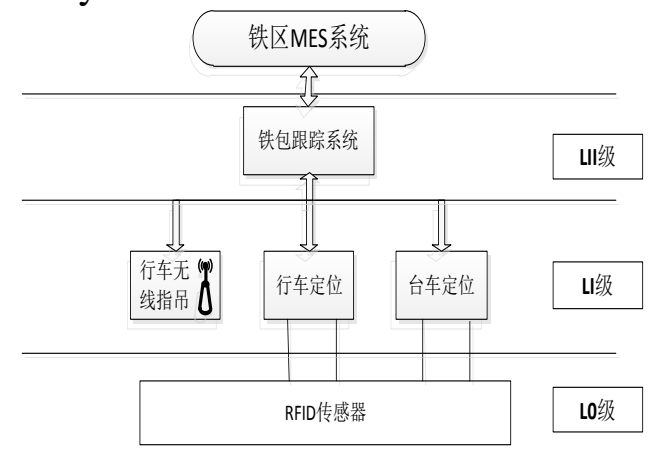

Fig. 1 the architecture diagram of Iron package tracking system

\section{System overview}

The data of Iron package tracking system comes from four parts: vehicle positioning information, car driving wireless positioning information, refers to the train operation instructions of hoisting module and the upper MES production plan data. The architecture diagram of iron package tracking system is shown in figure 1.Diagram, L0 level for the front-end data acquisition layer, mainly to complete data collection and conversion; LI level control layer, analysis L0 layer of real-time data to make the corresponding control management, such as vehicle refers to the orientation of crane, crane and trolley of iron package tracking control; LII level to system level, mainly to store and manage the data of LI layers', provide support for the upper management level, and provide the human-computer 
interaction interface. Through the iron area MES system integration platform function realizing standard of data transmission- -on and exchange between multiple LII level system and MES system.

\subsection{Operating principle of the system}

In order to achieve the iron ladle online and offline tracking, by positioning the vehicle and bogie to achieve iron ladle online production process tracking, to maintain the iron packages need to be repaired in the offline information entry, the complete track of iron package for the entire life cycle. The system is mainly composed of a driving trolley positioning module, positioning module, iron wire management module and animation simulation interface module modules. The vehicle positioning module and a trolley positioning module is the core part of iron ladle tracking system, the two positioning module based on RFID technology is a key point module.

\subsection{The trolley positioning module}

Position information acquisition process is as follows: the trolley at the site of the tapping position and driving lifting a side are respectively installed RFID identification device, when installing a molten iron trolley electronic label by installing a tapping RFID reader, electronic label reader queries received signal emitted by the signal reflection, the electronic tag response and will contain a trolley equipment information back to the reader, then the label information collected by the reader is processed through RS-485 transmitted to PLC controller. PLC combined with RFID electronic label information, trolley motor trolley on hot metal collection of the positive iron waterwheel location information to identify current passing and driving direction, then the PLC package trolley position information and other data to the LII grade iron package tracking system through RS-485 communication protocol, the system through the hot metal after treatment to identify current after the trolley contained in the iron, trolley, trolley bags, number, location and direction of travel, the trolley positioning structure is shown in figure 2.

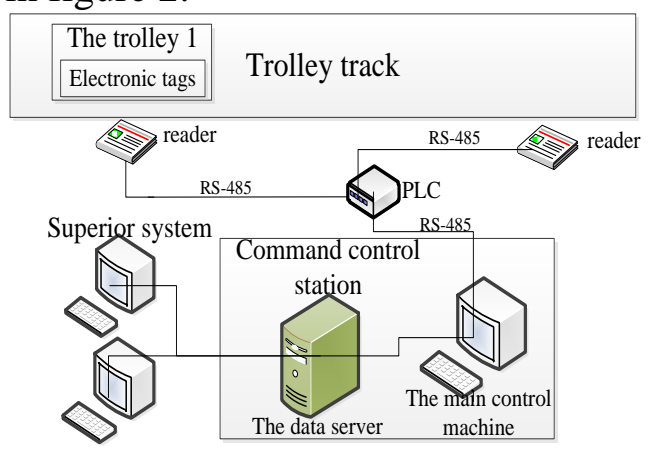

Fig. 2 A trolley positioning structure diagram

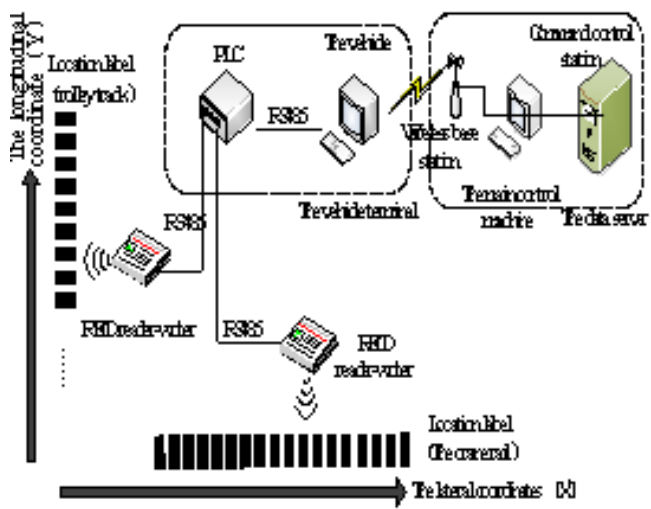

Fig. 3 Crane positioning structure diagram

\subsection{Vehicle positioning module}

Position information acquisition process is as follows: when vehicle running on the track with laying a tag and going through the electronic label position, installed in the driving of the RFID reader will serve as the acquisition device to collect coordinate information, read address coordinates and station code in electronic label of the position on the inside, the reader through RS-485 to transmit 
data to PLC, the position coordinates information of two readers will be encapsulated into data packets by PLC controller and via RS-485 to driving the vehicle terminal, It is sent to iron master tracking management room machine through the wireless LAN by the vehicle terminal system, main control computer analyze received data, judge the current iron ladle the operation state and sends driving lifting instruction, at the same time the data will be stored by the data server, other related department through the sharing of the data server to realize data resources. Driving positioning structure is shown in figure 3.

\section{The hardware design of the RFID reader}

The RFID reader structurally consist of three parts, the control module, the radio frequency module and the serial communication module, and the hardware design is presented in Fig 4. The control unit is the core of the control system, and C8051F340 is chosen as the control chip in the hardware design of the RFID reader. As the chosen radio frequency chip could independently complete the encoding and decoding specified by ISO18000-C protocol, CRC check sum and framing, there is not high requirements on the external microprocessor, and C8051F340 can satisfy the requirement of data acquisition, which can also reduce the cost of procurement of equipment. According to the working principle, radio frequency module can be divided into two parts, the transmitting channel and receiving channel. The radio frequency module has many functions, such as the generation of the radio frequency signal, filtering, modulation and demodulation, the processing of 18000-6C protocol, signal amplification, signal receiving and power detection and so on. And the radio frequency transceiver chip AS3992 is the core of radio-frequency circuit.In the design of the communication interface circuit, two different communication interfaces,RS-232 and RS-485 are used in order to satisfy the two kinds of real time operations after the data collection, the data transmission to PLC and the serial debugging between RFID reader and the PC machine.RS-485 is connected with the PLC controller for the data collection. And the RS-232 is connected with the PC machine for the data test.

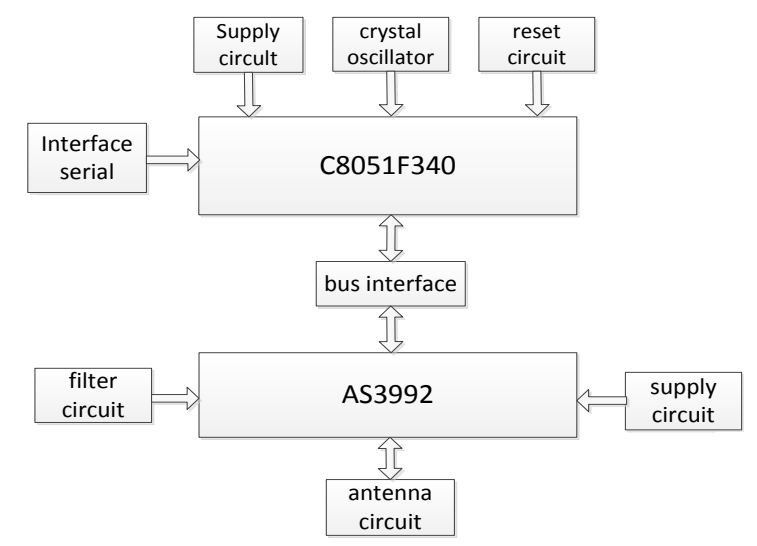

Fig. 4 the hardware design

\section{The network topology of the iron ladle tracking system}

There are three layers in the network topology of the iron ladle tracking system depending on the vehicle positioning, trolley positioning, the offline management module and the operational principle of RFID. The first layer is the data collection layer, which mainly collect the information of the statue of bridge crane, trolley and the station. The information of the statue of bridge crane is collected by the module of vehicle positioning, and then is uploaded to the car terminal packaged by PLC, and then is uploaded to the superior system after processing by the car terminal. The information of the statue of trolley is collected by the module of trolley positioning and then after being packaged by PLC, uploaded to the superior system by RS-485. The second layer is the system server layer, which mainly analyze the collected data, store the information in the server, provide data for the upper 
application and swap data with other systems. The third layer is the system application layer, which mainly realize the display of the tracing information and other related operations.

\section{Conclusions}

This system can satisfy the production needs and management needs of an iron and steel plant in Chongqing. The real time status of iron package is grasped timely, which improves the efficiency of iron ladle use, increases the production efficiency, reduces the error rate, improves the data accuracy and brings great economic benefits.

\section{Reference}

[1] Chen Chao. 2013. Study on the Positioning Technology Based on RFID. [D]Chen Du: University of Electronic Science and Technology.

[2] Liu Leilei. 2013. Design of Rail Vehicle Positioning System [D] Shen Yang: Shenyang University of Technology.

[3] Lee H.S., Murthy S.S., Haider S.W. 1996 Primary production scheduling at steelmaking industries [J]. IBM Journal of Research and Development, 40(2): 231-252.

[4] Stephens Andie. 1998. Integrated Scheduling of Steel Production [J]. Asia Steel: 150-153. 